\title{
Proteomic analysis of mammalian sperm cells identifies new components of the centrosome
}

\author{
Elif N. Firat-Karalar ${ }^{1,3}$, Joshua Sante ${ }^{1,4}$, Sarah Elliott ${ }^{1}$, and Tim Stearns ${ }^{1,2}$ \\ ${ }^{1}$ Department of Biology, Stanford University, Stanford, CA 94305-5020, USA \\ ${ }^{2}$ Department of Genetics, Stanford School of Medicine, Stanford, CA 94305-5120, USA \\ ${ }^{3}$ Current address: Department of Molecular Biology and Genetics, Koç University, \\ Istanbul, 34450, Turkey \\ ${ }^{4}$ Current address: Division of Pulmonary and Critical Care, Stanford School of Medicine, \\ Palo Alto, CA 94304, USA \\ Corresponding Author: \\ Tim Stearns \\ Department of Biology \\ Stanford University \\ Tel: (650) 725-6934 \\ Fax: (650) 724-9945 \\ E-mail: stearns@stanford.edu
}




\section{Abstract}

Centrioles are evolutionarily conserved microtubule-based structures at the core of the animal centrosome that are essential for nucleating the axoneme of cilia. We hypothesized that centriole proteins have been under-represented in proteomic studies of the centrosome, because of the larger amount of pericentriolar material making up the centrosome. In this study, we have overcome this problem by determining the centriolar proteome of mammalian sperm cells, which have a pair of centrioles but little pericentriolar material. Mass spectrometry of sperm centrioles identifies known components of centrioles and many previously uncharacterized candidate centriole proteins. Assessment of localization of a subset of these candidates in cultured cells identified CCDC113, CCDC96, C4orf47, CCDC38, C7orf31, CCDC146, CCDC81 and CCDC116 as centrosome-associated proteins. We examined the highly conserved protein CCDC113 further and found that it is a component of centriolar satellites, is in a complex with the satellite proteins HAP1 and PCM1, and functions in primary cilium formation. 


\section{Introduction}

The centriole is a microtubule-based structure conserved in all eukaryotes that make cilia (Hodges et al., 2010; Carvalho-Santos et al., 2011). The centriole has a unique nine-fold symmetry in which compound microtubules (doublets and triplets) are radially arranged in a cylinder of about $500 \mathrm{~nm}$ in length and $275 \mathrm{~nm}$ in diameter (Chretien et al., 1997). Centrioles organize the centrosome by recruiting a matrix of associated pericentriolar material (PCM) that supports microtubule nucleation and polymerization and organization (Luders and Stearns, 2007). Importantly, centrioles nucleate the axonemal microtubules of the cilium thus imparting their unique structure on the axoneme (Fisch and Dupuis-Williams, 2011).

A combination of proteomic, bioinformatic and genomic approaches (Ostrowski et al., 2002; Avidor-Reiss et al., 2004; Li et al., 2004; Keller et al., 2005; Kilburn et al., 2007; McClintock et al., 2008; Keller et al., 2009; Jakobsen et al., 2011; Hoh et al., 2012; Ishikawa et al., 2012) has identified many centrosome and cilium proteins. The centriole proteomes of Chlamydomonas (Keller et al., 2005) and Tetrahymena (Kilburn et al., 2007) have been analyzed, but direct proteomic analysis of mammalian centrioles has not been reported. Morphological analysis of centrioles from several species ( $\mathrm{Li}$ et al., 2012; Guichard et al., 2013) reveals conserved structural features, and, other than the nine-fold symmetry of centrioles, which is dictated by the structure of SASS6 (Gonczy, 2012), these features have no known molecular basis. For example, cryotomography and 3D-reconstruction studies of Chlamydomonas (Li et al., 2012) and Trichonympha centrioles (Guichard et al., 2013) show non-tubulin densities associated with the lumen of microtubule doublets and triplets, and with the linker between them.

To identify centriole proteins, we exploited mammalian sperm cells, which have centrioles that have lost most associated PCM and the capacity to nucleate microtubules during spermatogenesis (Manandhar et al., 2005). A mature mammalian sperm typically has two orthogonal centrioles in the midpiece between head and tail, a well-defined proximal centriole, and a degenerated distal centriole that nucleates the flagellar axoneme (Manandhar et al., 2000). Centrioles are paternally inherited in most vertebrates (Simerly et al., 1995), and once introduced into the egg by fertilization, the centrioles recruit PCM to form the centrosome (Stearns and Kirschner, 1994). 
Therefore, analysis of sperm centrioles might be informative in identifying components required for PCM organization and for cilium formation.

In this study, we isolated centrioles from bovine sperm and identified 364 proteins by mass spectrometry, of which we confirmed 8 as previously uncharacterized centrosome-localizing proteins. One of these, CCDC113, is a component of centriolar satellites that is highly conserved and functions in cilium formation.

\section{Results and Discussion}

We developed a protocol for enriching for sperm centrioles based on previous studies (Olson et al., 1976; Kleve and Clark, 1980). The centriole enrichment protocol was guided by the specialized structure of mature sperm cells, which consists of a head (acrosome and nucleus), a midpiece (proximal and distal centrioles, mitochondria) and a tail (axoneme) (Palermo et al., 1997). We chose bovine sperm as the starting material for the centriole enrichment; Fig.1A shows these cells stained with antibodies against the centriole marker centrin, the axoneme marker tubulin and the DNA stain DAPI. Bovine sperm were sonicated to separate heads from tails, and sedimented through a glycerol cushion, isolating the tails in the supernatant (Fig.1B). Successful fractionation was confirmed by immunostaining (Fig.1C) and immunoblotting (Fig.1D) for the nuclear marker histone H3 (heads fraction), and centrin (tail fraction). The tail fractions were sequentially extracted with buffers of increasing stringency to enrich for centrioles (Olson et al., 1976). Separation of centrioles from midpiece mitochondria and the bulk of the axoneme was confirmed by immunoblotting for the mitochondria marker MtHsp70, (first extraction fraction), centrin (second extraction fraction) and the axoneme marker tubulin (third extraction fraction) (Fig.1E). Analysis of the centriole-enriched fractions (ext\#2) from two independent experiments by LC-MS mass spectrometry yielded a candidate sperm centriole proteome of 364 proteins (Table S1).

To assess the sperm centriole proteome candidates, all 364 proteins were categorized by gene ontology molecular compartment, and cross-referenced with proteomic, bioinformatic and genomic studies of centrosomes and cilia (Fig.1F,Table S2). $60 \%$ of the sperm centriole candidate proteins were previously identified in one or more of these studies, and 13 proteins were previously shown to localize to the 
centrosome (Table S2), including the centriole components centrin 1 (Hart et al., 1999) and POC1 (Keller et al., 2009). Few proteins were annotated as having a molecular compartment consistent with them being contaminants (Fig.1F). In contrast, our analysis of the data from the previous studies of the mammalian sperm tail proteome (Baker et al., 2013), which did not enrich for the centriole fraction, revealed that only $11 \%$ of the identified proteins corresponded to previously identified centrosome and cilium proteins. Interestingly, $19 \%$ of the candidate proteins from our dataset did not have an associated molecular compartment, and most of these proteins are previously uncharacterized.

A subset of candidate proteins were selected for further characterization in cultured human cells, based on several criteria. CCDC96, CCDC113, CCDC81, LRRC34, LRRC48, WDR65 and C4orf47 were identified by one or more systematic previous studies of centrosomes and cilia; CCDC146, C4orf47, CCDC96 and CCDC113 are conserved in most ciliated eukaryotes; EFCAB1, WDR65, LRRC34, LRRC48, TTC18, CCDC38, CCDC81, CCDC108 and CCDC116 contain domains or structural motifs found in proteins associated with centrosome or cilia. In addition to the above, C7orf31 and C9ORF116 did not fall any of these criteria and were chosen randomly from among the uncharacterized proteins. Publically available expression data showed that these proteins varied in expression pattern, from wide distribution in most tissues to highly-restricted to the testis (Fig.S1B). The ability of these proteins to localize to the centrosome was assayed by cloning the corresponding cDNAs with $\mathrm{N}$ - and/or Cterminal GFP tags and expression by transient transfection in HeLa cells, which do not form a primary cilium, and RPE1 cells, which were induced to form a primary cilium by serum starvation (Fig.S1B). CCDC146 localization was determined by immunostaining with antibody against CCDC146. Eight of the 15 tagged candidate proteins localized at or near the centrosome, as assessed by localization relative to gamma-tubulin or polyglutamylated tubulin, as indicated (Fig.2). Most of the proteins largely colocalized with gamma-tubulin; the exceptions were CCDC113, which localized to punctate structures resembling centriolar satellites, and CCDC146, which localized to only one centriole (Fig.2), identified as the mother centriole by costaining with CEP164 (Fig.S1A). The remaining 7 of the 15 tagged proteins did not localize to the centrosome (Fig.S1B). 
We chose CCDC113, for further study, based on its putative localization to centriolar satellites. Centriolar satellites are linked to several centrosome-related processes including centrosome and cilium protein trafficking, ciliogenesis, and centriole duplication (Firat-Karalar et al., 2014), and implicated in ciliopathies (Barenz et al., 2011). CCDC113 is related to one of the other centrosome-localizing proteins, CCDC96 (Fig.S2A) and orthologs of both proteins are present in chordates, deuterostomes, schistosomes, Trichoplax and Chlamydomonas, but not in C. elegans (Fig.S2A). RTPCR experiments using RNA from RPE1 cells (Fig.3A) and immunoblotting with an antibody against CCDC113 (Fig.3B) showed that CCDC113 is expressed in RPE1, U2OS and HeLa cells.

The punctate pericentrosomal distribution of satellite proteins is dependent on the satellite protein PCM1 and a centrosomally-focused microtubule array (Kim et al., 2004; Kim et al., 2008; Lopes et al., 2011). CCDC113-GFP localized to a subset of PCM1-positive puncta in RPE1 cells (Fig.3B,C), and these CCDC113-GFP/PCM1positive puncta dispersed upon depolymerization of microtubules (Fig.3C), as expected for centriolar satellites. Moreover, depletion of PCM1 caused loss of CCDC113-GFP satellite localization (Fig.3D). In both cases loss of CCDC113-GFP localization to satellites was accompanied by localization of the protein to the centrosome, as observed for some other satellite components (Stowe et al., 2012). These results identify CCDC113-GFP as a new component of centriolar satellites.

A high-throughput two-hybrid screen (Stelzl et al., 2005) identified an interaction between CCDC113 and the centriolar satellite protein HAP1 (Engelender et al., 1997; Keryer et al., 2011). We validated this interaction in co-immunoprecipitation experiments in HEK293T cells in which CCDC113 interacted with both HAP1 and PCM1 (Fig.4A, Fig.S2B,C). Moreover, CCDC113-GFP colocalized with HAP1 at centriolar satellites (Fig.4B). HAP1 forms a complex with PCM1 and huntingtin and regulates ciliogenesis (Engelender et al., 1997; Keryer et al., 2011). We tested whether CCDC113 also is required for efficient ciliogenesis. CDC113 was depleted from RPE1 cells by infection with a lentivirus expressing short hairpin RNA (shRNA) targeting CCDC113. Efficient depletion of CCDC113 was observed $7 \mathrm{~d}$ after infection, as demonstrated by immunoblotting for CCDC113 (Fig.S2D). Depletion of CCDC113 significantly reduced 
cilium formation in serum-starved RPE1 cells, and this phenotype was rescued by expression of GFP-tagged mouse CCDC113 (Fig.3C,D). The ciliogenesis phenotype was not due to a defect in cell cycle progression, since both control and CCDC113depleted cells had similar cell cycle profiles as analyzed by flow cytometry (Fig.S2E). As with HAP1 (Keryer et al., 2011), depletion of CCDC113 caused dispersal of PCM1positive satellites throughout the cytoplasm relative to control cells in which satellites were focused around the centrosome (Fig.4E). These phenotypes suggest that CCDC113 functions in ciliogenesis.

In this report, we used proteomic analysis of centrioles of a differentiated cell type, the mammalian sperm, to identify putative components of the centrosome in general. The combination of the unique properties of the sperm centrioles with the sperm centriole enrichment protocol yielded a particularly specific dataset of proteins that localize to the centrosome. Many of the most well characterized centrosome proteins are PCM components (Jakobsen et al., 2011). Thus, this dataset is likely to be particularly useful for studies of the centriole, about which little is known with respect to the relationship between structure (Li et al., 2012; Guichard et al., 2013) and specific proteins.

Eight of the 15 previously uncharacterized proteins tested in this study localize at or near the centrosome in cycling cells as GFP fusion proteins, validating the original assumption that the sperm centrioles have components in common with the centrosome. Except for the mother centriole protein CCDC146, most of the GFP-tagged proteins localized to a larger centrosome domain, mostly overlapping with the PCM marker gamma-tubulin. This might reflect situations such as with ODF2, which is part of morphologically distinct structures at the centrosome and in the sperm midpiece (Brohmann et al., 1997), or might be due to overexpression of the proteins, which can result in a broader distribution of centriolar proteins. Given the highly specialized structure and function of the sperm, we expected that the sperm centriole proteome would also include proteins specific to sperm. Indeed, several of these proteins have EST expression profiles that are highly specific for testis (Fig.S1B). For such proteins, the ability of the protein to localize to the centrosome suggests that the protein or 
structure that it interacts with at the centrosome is common between sperm centrioles and cycling cell centrosomes.

Focusing on one of the sperm centriole candidates, we identified CCDC113 as a new component of centriolar satellites that is important for efficient ciliogenesis. We note that the pattern of evolutionary conservation of CCDC113 is similar to that for the satellite proteins BBS4 (Kim et al., 2004) and CEP290 (Kim et al., 2008), but not to that for PCM1 (Hodges et al., 2010). This suggests that the interaction of CCDC113 with satellites is likely to represent modulation of CCDC113 by satellites, rather than function of satellites. Consistent with this hypothesis, we found that elimination of satellites by PCM1 depletion results in relocalization of $C C D C 113$ to the centrosome, suggesting that satellites sequester CCDC113 from the centrosome. Sperm lack the complement of satellites typical of most somatic cells, but a recent study (Hall et al., 2013) demonstrated that the satellite protein AZI1 is required for sperm motility by regulating protein trafficking in sperm cells (Hall et al., 2013). CCDC113 and AZI1 might represent a class of proteins that have similar functions in sperm cells and somatic cells, but for which that function is regulated by association with satellites in somatic cells. 


\section{Materials and Methods}

\section{Cell culture and transfection}

HeLa and HEK293T cells were grown in DMEM supplemented with $10 \%$ fetal bovine serum (FBS; Atlanta Biologicals, Lawrenceville, GA). RPE1 cells were grown in DMEM/F12 50/50 supplemented with 10\% FBS. Bovine sperm (Bovine Elite, College Station, TX) were used for centriole enrichment. RPE1 and HeLa cells were transfected using Lipofectamine LTX (Invitrogen). HEK293T cells were transfected using $1 \mu \mathrm{g} / \mu \mathrm{l}$ polyethylenimine, MW $25 \mathrm{kDa}$ (Sigma-Aldrich, St. Louis, MO).

\section{Sperm centriole enrichment}

Bovine sperm were rinsed with PBS and sonicated to separate heads from tails, and sedimented through a $30 \%$ glycerol cushion to isolate the tails in the supernatant from the heads in the pellet. The tail fractions were sequentially extracted at $4{ }^{\circ} \mathrm{C}$ for $1 \mathrm{~h}$ with extraction buffer 1 (50 mM Tris pH 8, 2 mM EGTA, 2 mM EDTA, 500 mM NaCl, 1\% NP$40,0.1 \% \beta$-mercaptoethanol, $1 \mathrm{mM}$ DTT, inhibitors), extraction buffer 2 (50 mM Tris $\mathrm{pH}$ 8, $600 \mathrm{mM} \mathrm{KSCN}, 2 \mathrm{mM}$ DTT, inhibitors) and extraction buffer 3 (50 mM Tris pH 8, 4 M urea, inhibitors). The supernatant after each extraction was precipitated with TCA.

\section{Plasmids}

The GenBank/EMBL/DDBJ accession numbers and sources of all the cDNAs used in this study are described in Fig.S1. The CCDC96 ORF was amplified by PCR and cloned into peGFP-N1. The ORFs of the remaining genes were amplified by PCR and cloned into pDONR221 (Invitrogen). Subsequent Gateway recombination reactions using pCS2+6xMyc DEST and pcDNA-DEST47 were used to generate Myc- and/or GFPtagged proteins.

\section{RT-PCR and Immunoprecipitation experiments}

Total RNA was isolated from RPE1 cells using an RNeasy kit (Qiagen, Chatsworth, CA) and RT-PCR was performed using a cDNA synthesis kit (Invitrogen, San Diego, CA). Primers used were 5'CGGAGCCTCTTATTCACTGC3' and 
5'AGCAAGCTTCACAAGGCAAT3'. Co-immunoprecipitation experiments using HEK293T cells were done as described (Firat-Karalar et al., 2014).

\section{shRNA and rescue experiments}

CCDC113 shRNA oligos (nucleotides 300-321-5'ATAGCAGAGATGTCCTTAAA3') were cloned into pLKO.1. CCDC113 depletion was carried out by infection of cells with shRNA-expressing lentivirus as described (Mahjoub et al., 2010). PCM1 was depleted using an siRNA as described previously (Firat-Karalar et al., 2014). For rescue experiments, pools of RPE-1 cells stably expressing GFP (RPE-1::GFP) or mouse CCDC113-GFP (RPE-1:: mCCDC113-GFP) were infected with lentivirus expressing empty vector or shRNA targeting CCDC113. Cells were serum starved for $48 \mathrm{~h}$, fixed and stained on day 7 for the subsequent ciliogenesis assays.

\section{Antibodies}

Anti-CCDC146 antibody was obtained by immunizing rabbits (Cocalico Biologicals, Reamstown, PA) with GST-tagged CCDC146 (aminoacids 1-461). The antibody was affinity purified against MBP-CCDC146 (1-461) and used at $0.5 \mathrm{ug} / \mathrm{ml}$ for immunofluorescence. Other antibodies used are described in Fig.S1C.

\section{Mass spectrometry}

The centriole fraction was analyzed using LCq-Deca-XPPlus mass spectrometer (Thermo Scientific) at the Stanford Mass Spectrometry Laboratory. The database was composed of B.taurus protein sequences archived in the National Center for Biotechnology Information. Proteins were identified on the basis of at least two peptide matches with a probability $>95 \%$. The human orthologs of the $B$. taurus proteins for which annotation was not available were identified by reciprocal Blast analysis.

\section{Immunofluorescence and microscopy}

For immunofluorescence experiments, cells were grown on coverslips, fixed in methanol, processed for indirect immunofluorescence and imaged as described (FiratKaralar et al., 2014). Sperm cells were dried on coverslips and processed for immunofluorescence as described for cell lines. 


\section{Acknowledgements}

This work was supported by NRSA Grant 5F32GM106620 to ENF and NIH Grant R01 GM52022 to TS. 


\section{Figure Legends}

\section{Figure 1. Centriole enrichment protocol from bovine sperm cells}

(A) Bovine sperm, fixed and stained with antibodies against centrin and $\alpha$-tubulin. DNA was stained with DAPI. Scale bar, $5 \mu \mathrm{m}$. (B) Intact sperm, isolated heads and tails imaged by phase microscopy. Scale bar, $10 \mu \mathrm{m}$. (C) Isolated tails fixed and stained for centrin and $\alpha$-tubulin. Scale bar, $5 \mu \mathrm{m}$. (D) Extracts of intact sperm, isolated heads and tails blotted for centrioles, Histone $\mathrm{H} 3$ and $\alpha$-tubulin. Extracts from each extraction step were blotted for centrin, $\alpha$-tubulin and MtHsp70.All insets 4x.(E) Categorization of sperm centriole candidate proteins according to gene ontology molecular compartment.

\section{Figure 2. Localization of new centrosome proteins}

(A) HeLa cells were transfected with the indicated constructs. $24 \mathrm{~h}$ after transfection, cells were fixed and stained with anti-GFP antibody or with anti-CCDC146 antibody. Centrosomes were stained with anti- $\gamma$-tubulin antibody and DNA was stained with DAPI. (B) RPE1 cells were transfected with the indicated constructs, serum-starved for $24 \mathrm{~h}$, fixed and stained with anti-GFP antibody or with anti-CCDC146 antibody. The primary cilium and centrioles were stained with polyglutamylated tubulin antibody and DNA was stained with DAPI. Scale bar, $10 \mu \mathrm{m}$, all insets $4 \mathrm{x}$.

\section{Figure 3. CCDC113 expression and localization in cultured human cell lines.}

(A) RT-PCR analysis of RNA from RPE1 cells with a pair of CCDC113-specific primers with (+) or without (-) the addition of reverse transcriptase. Positive control is PCR amplification of CCDC113 gene with these primers. (B) Extracts from RPE1, HeLa and U2OS cells were blotted for CCDC113 and p38 (loading control). (C) RPE1 cells expressing CCDC113-GFP were treated with DMSO or $10 \mu \mathrm{g} / \mathrm{ml}$ nocodazole, fixed, stained for GFP, PCM1 and $\gamma$-tubulin. (D) RPE1 cells expressing CCDC113-GFP were fixed $48 \mathrm{~h}$ after transfection with control or PCM1 siRNA, and stained for GFP, PCM1 and $\gamma$-tubulin. Scale bars, $10 \mu \mathrm{m}$.

Figure 4: CCDC113 functions in ciliogenesis 
(A) Co-immunoprecipitation of CCDC113-GFP, tomato-PCM1(td-PCM1) and HAP1-Myc after co-expression in HEK293T cells. Complexes were immunoprecipitated with antiGFP antibody and co-precipitated proteins were detected with anti-GFP, anti-Myc and anti-PCM1 antibodies. (B) RPE1 cells expressing HAP1-Myc and CCDC113-GFP were stained with anti-Myc, and anti-GFP antibodies. DNA was stained with DAPI. (C) RPE1 cells were fixed $7 d$ after infection with virus expressing empty vector or CCDC113 shRNA, and percentage of ciliogenesis was determined by staining with polyglutamylated tubulin and DAPI. (D) Quantification of C. $n \geq 100$ cells per experiment. ${ }^{* *} p<0.01,{ }^{*} p<0.05$ (E) Control and CCDC113-depleted RPE1 cells were fixed, stained for PCM1. DNA was stained with DAPI. Scale bars, $10 \mu \mathrm{m}$, all insets $4 \mathrm{x}$. 


\section{References}

Avidor-Reiss, T., Maer, A. M., Koundakjian, E., Polyanovsky, A., Keil, T., Subramaniam, S. and Zuker, C. S. (2004). Decoding cilia function: defining specialized genes required for compartmentalized cilia biogenesis. Cell 117, 527-539.

Baker, M. A., Naumovski, N., Hetherington, L., Weinberg, A., Velkov, T. and Aitken, R. J. (2013). Head and flagella subcompartmental proteomic analysis of human spermatozoa. Proteomics 13, 61-74.

Barenz, F., Mayilo, D. and Gruss, O. J. (2011). Centriolar satellites: busy orbits around the centrosome. Eur J Cell Biol 90, 983-989.

Brohmann, H., Pinnecke, S. and Hoyer-Fender, S. (1997). Identification and characterization of new cDNAs encoding outer dense fiber proteins of rat sperm. J Biol Chem 272, 10327-10332.

Carvalho-Santos, Z., Azimzadeh, J., Pereira-Leal, J. B. and Bettencourt-Dias, M. (2011). Evolution: Tracing the origins of centrioles, cilia, and flagella. J Cell Biol 194, 165-175.

Chretien, D., Buendia, B., Fuller, S. D. and Karsenti, E. (1997). Reconstruction of the centrosome cycle from cryoelectron micrographs. J Struct Biol 120, 117-133.

Engelender, S., Sharp, A. H., Colomer, V., Tokito, M. K., Lanahan, A., Worley, P., Holzbaur, E. L. and Ross, C. A. (1997). Huntingtin-associated protein 1 (HAP1) interacts with the p150Glued subunit of dynactin. Hum Mol Genet 6, 2205-2212. Firat-Karalar, E. N., Rauniyar, N., Yates, J. R., 3rd and Stearns, T. (2014). Proximity interactions among centrosome components identify regulators of centriole duplication. Curr Biol 24, 664-670.

Fisch, C. and Dupuis-Williams, P. (2011). Ultrastructure of cilia and flagella - back to the future! Biol Cell 103, 249-270.

Gonczy, P. (2012). Towards a molecular architecture of centriole assembly. Nat Rev Mol Cell Biol 13, 425-435.

Guichard, P., Hachet, V., Majubu, N., Neves, A., Demurtas, D., Olieric, N., Fluckiger, I., Yamada, A., Kihara, K., Nishida, Y. et al. (2013). Native architecture of 
the centriole proximal region reveals features underlying its 9-fold radial symmetry. Curr Biol 23, 1620-1628.

Hall, E. A., Keighren, M., Ford, M. J., Davey, T., Jarman, A. P., Smith, L. B., Jackson, I. J. and Mill, P. (2013). Acute versus chronic loss of mammalian Azi1/Cep131 results in distinct ciliary phenotypes. PLoS Genet 9, e1003928.

Hart, P. E., Glantz, J. N., Orth, J. D., Poynter, G. M. and Salisbury, J. L. (1999). Testis-specific murine centrin, Cetn1: genomic characterization and evidence for retroposition of a gene encoding a centrosome protein. Genomics 60, 111-120. Hodges, M. E., Scheumann, N., Wickstead, B., Langdale, J. A. and Gull, K. (2010). Reconstructing the evolutionary history of the centriole from protein components. J Cell Sci 123, 1407-1413.

Hoh, R. A., Stowe, T. R., Turk, E. and Stearns, T. (2012). Transcriptional program of ciliated epithelial cells reveals new cilium and centrosome components and links to human disease. PLoS One 7, e52166.

Ishikawa, H., Thompson, J., Yates, J. R., 3rd and Marshall, W. F. (2012). Proteomic analysis of mammalian primary cilia. Curr Biol 22, 414-419.

Jakobsen, L., Vanselow, K., Skogs, M., Toyoda, Y., Lundberg, E., Poser, I., Falkenby, L. G., Bennetzen, M., Westendorf, J., Nigg, E. A. et al. (2011). Novel asymmetrically localizing components of human centrosomes identified by complementary proteomics methods. EMBO J 30, 1520-1535.

Keller, L. C., Romijn, E. P., Zamora, I., Yates, J. R., 3rd and Marshall, W. F. (2005). Proteomic analysis of isolated chlamydomonas centrioles reveals orthologs of ciliarydisease genes. Curr Biol 15, 1090-1098.

Keller, L. C., Geimer, S., Romijn, E., Yates, J., 3rd, Zamora, I. and Marshall, W. F. (2009). Molecular architecture of the centriole proteome: the conserved WD40 domain protein POC1 is required for centriole duplication and length control. Mol Biol Cell 20, 1150-1166.

Keryer, G., Pineda, J. R., Liot, G., Kim, J., Dietrich, P., Benstaali, C., Smith, K., Cordelieres, F. P., Spassky, N., Ferrante, R. J. et al. (2011). Ciliogenesis is regulated by a huntingtin-HAP1-PCM1 pathway and is altered in Huntington disease. $J$ Clin Invest 121, $4372-4382$. 
Kilburn, C. L., Pearson, C. G., Romijn, E. P., Meehl, J. B., Giddings, T. H., Jr., Culver, B. P., Yates, J. R., 3rd and Winey, M. (2007). New Tetrahymena basal body protein components identify basal body domain structure. J Cell Biol 178, 905-912.

Kim, J., Krishnaswami, S. R. and Gleeson, J. G. (2008). CEP290 interacts with the centriolar satellite component PCM-1 and is required for Rab8 localization to the primary cilium. Hum Mol Genet 17, 3796-3805.

Kim, J. C., Badano, J. L., Sibold, S., Esmail, M. A., Hill, J., Hoskins, B. E., Leitch, C. C., Venner, K., Ansley, S. J., Ross, A. J. et al. (2004). The Bardet-Biedl protein BBS4 targets cargo to the pericentriolar region and is required for microtubule anchoring and cell cycle progression. Nat Genet 36, 462-470.

Kleve, M. G. and Clark, W. H., Jr. (1980). Association of actin with sperm centrioles: isolation of centriolar complexes and immunofluorescent localization of actin. J Cell Biol 86, 87-95.

Li, J. B., Gerdes, J. M., Haycraft, C. J., Fan, Y., Teslovich, T. M., May-Simera, H., Li, H., Blacque, O. E., Li, L., Leitch, C. C. et al. (2004). Comparative genomics identifies a flagellar and basal body proteome that includes the BBS5 human disease gene. Cell 117, 541-552.

Li, S., Fernandez, J. J., Marshall, W. F. and Agard, D. A. (2012). Three-dimensional structure of basal body triplet revealed by electron cryo-tomography. EMBO J 31, 552562.

Lopes, C. A., Prosser, S. L., Romio, L., Hirst, R. A., O'Callaghan, C., Woolf, A. S. and Fry, A. M. (2011). Centriolar satellites are assembly points for proteins implicated in human ciliopathies, including oral-facial-digital syndrome 1. J Cell Sci 124, 600-612. Luders, J. and Stearns, T. (2007). Microtubule-organizing centres: a re-evaluation. Nat Rev Mol Cell Biol 8, 161-167.

Mahjoub, M. R., Xie, Z. and Stearns, T. (2010). Cep120 is asymmetrically localized to the daughter centriole and is essential for centriole assembly. J Cell Biol 191, 331-346. Manandhar, G., Simerly, C. and Schatten, G. (2000). Highly degenerated distal centrioles in rhesus and human spermatozoa. Hum Reprod 15, 256-263.

Manandhar, G., Schatten, H. and Sutovsky, P. (2005). Centrosome reduction during gametogenesis and its significance. Biol Reprod 72, 2-13. 
McClintock, T. S., Glasser, C. E., Bose, S. C. and Bergman, D. A. (2008). Tissue expression patterns identify mouse cilia genes. Physiol Genomics 32, 198-206.

Olson, G. E., Hamilton, D. W. and Fawcett, D. W. (1976). Isolation and characterization of the fibrous sheath of rat epididymal spermatozoa. Biol Reprod 14, 517-530.

Ostrowski, L. E., Blackburn, K., Radde, K. M., Moyer, M. B., Schlatzer, D. M., Moseley, A. and Boucher, R. C. (2002). A proteomic analysis of human cilia: identification of novel components. Mol Cell Proteomics 1, 451-465.

Palermo, G. D., Colombero, L. T. and Rosenwaks, Z. (1997). The human sperm centrosome is responsible for normal syngamy and early embryonic development. Rev Reprod 2, 19-27.

Simerly, C., Wu, G. J., Zoran, S., Ord, T., Rawlins, R., Jones, J., Navara, C., Gerrity, M., Rinehart, J., Binor, Z. et al. (1995). The paternal inheritance of the centrosome, the cell's microtubule-organizing center, in humans, and the implications for infertility. Nat Med 1, 47-52.

Stearns, T. and Kirschner, M. (1994). In vitro reconstitution of centrosome assembly and function: the central role of gamma-tubulin. Cell 76, 623-637.

Stelzl, U., Worm, U., Lalowski, M., Haenig, C., Brembeck, F. H., Goehler, H., Stroedicke, M., Zenkner, M., Schoenherr, A., Koeppen, S. et al. (2005). A human protein-protein interaction network: a resource for annotating the proteome. Cell 122, 957-968.

Stowe, T. R., Wilkinson, C. J., Iqbal, A. and Stearns, T. (2012). The centriolar satellite proteins Cep72 and Cep290 interact and are required for recruitment of BBS proteins to the cilium. Mol Biol Cell 23, 3322-3335. 


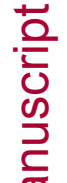

Figum 1

A E

ceptrin

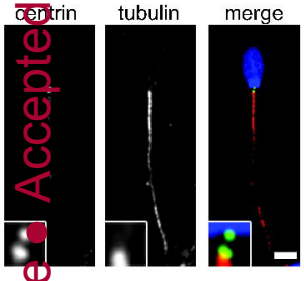

C

\section{certtrin}

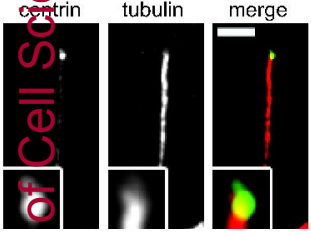

B
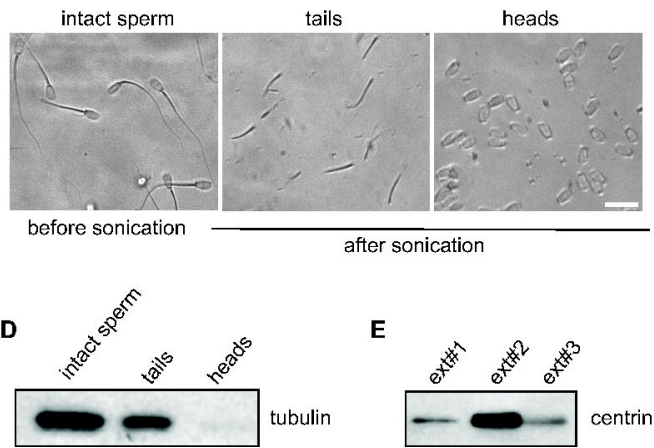

centrin

histone $\mathrm{H} 3$

E
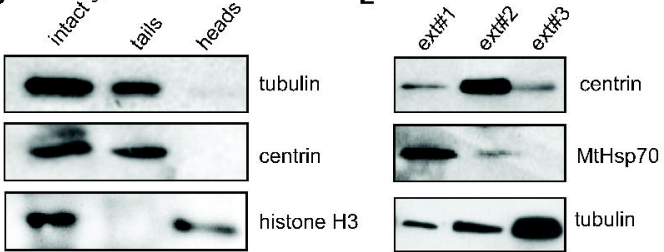
tubulin

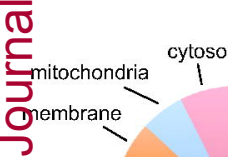

nucleus 


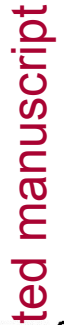

Figitire 3

A (1)

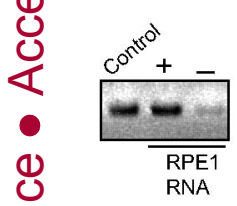

B

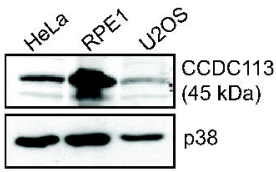

c $\frac{\overline{1}}{\mathrm{~N}}$

DN

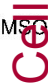

\section{CCDC113-}
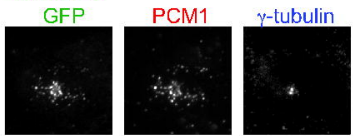

merge

DAPI

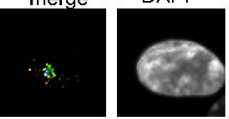

nocodazole

ธ
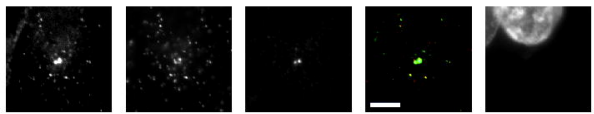

CCDC113-

욱
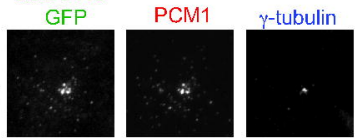

merge

DAPI
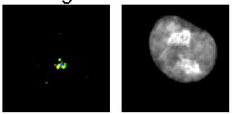

siPCM1
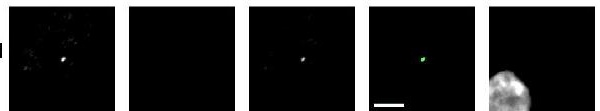


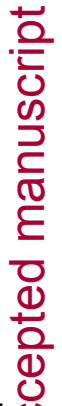

Figuibe 4

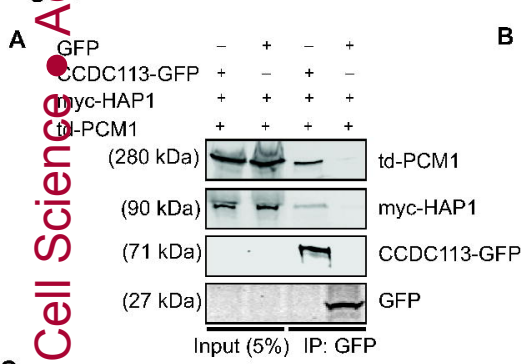

C

4

glut.

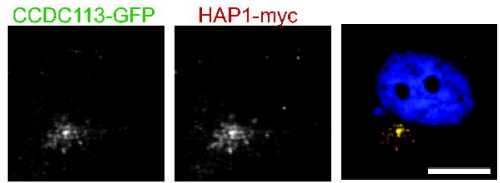

तั

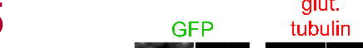

